PROCEEDINGS OF THE

AMERICAN MATHEMATICAL SOCIETY

Volume 126, Number 7, July 1998, Pages 1941-1944

S 0002-9939(98)04453-0

\title{
A NEW PROOF OF THE SOLOMON-TITS THEOREM
}

\author{
CHAO KU
}

(Communicated by Ronald M. Solomon)

\begin{abstract}
We give a new proof of the Solomon-Tits Theorem which asserts that the Tits building of a finite group of Lie type has the homotopy type of a bouquet of spheres.
\end{abstract}

\section{INTRODUCTION}

Let $G$ be a finite group of Lie type of rank $n \geqslant 3,(G, B, N, S)$ a Tits system for $G, H=B \cap N, B=U H$ where $U=O_{p}(B)$ and $p$ is the defining prime for $G$. $\mathbf{F}=\left\{G_{i} ; 1 \leqslant i \leqslant n\right\}$ is the set of maximal parabolics of $G$ over $B$ and $\mathcal{B}=\mathcal{C}(G, \mathbf{F})$ the simplicial complex whose vertex set is the union of coset spaces $G / G_{i}, i \in I$, and whose simplices are collection of vertices with nonempty intersection. $\mathcal{B}$ is called the Tits building of $G$. The following theorem is well known:

Theorem (Solomon-Tits). The Tits building $\mathcal{B}$ has the homotopy type of a wedge of $|U|(n-1)$-spheres.

In his seminar paper [S] Solomon first mentioned the theorem and sketched a proof. Later Garland ([G]) gave a proof which is essentially the same as Solomon's, and Curtis and Lehrer ([CL]) gave a proof of a related result based on the computation of the $\mathbb{Q} G$-endomorphism algebra of $H_{*}(\mathcal{B})$. We provide a new proof here which should help to better understand the structure of the Tits building. Namely, $\mathcal{B}$ is the union of the $|U|$ apartments containing a fixed chamber, each having the homotopy type of an $(n-1)$-sphere as it is a Coxeter complex. We show that the intersection of any $k(k \geqslant 2)$ of these subcomplexes is contractible. Then it follows from an easy lemma in topology that $\mathcal{B}$ has the homotopy type of a wedge of these spheres.

\section{Preliminary lemmas}

Let us begin by recording a well known lemma from topology.

Lemma 1. Suppose $X$ is a simplicial complex and $X_{1}, X_{2}, \ldots, X_{m}, m>1$, are subcomplexes of $X$ with $X=\bigcup_{1 \leqslant i \leqslant m} X_{i}$ such that for any $1 \leqslant i_{1}<i_{2}<\cdots<$ $i_{k} \leqslant m, k>1$, the set $\bigcap_{1 \leqslant j \leqslant k} X_{i_{j}}$ is contractible. Then $X$ has the homotopy type of $X_{1} \vee X_{2} \vee \cdots \vee X_{m}$.

Received by the editors December 19, 1996

1991 Mathematics Subject Classification. Primary 20E42.

(C)1998 American Mathematical Society 
Proof: By induction. Note that if $B$ is a contractible subcomplex of a simplicial complex $A$, then the quotient map $q: A \rightarrow A / B$ is a homotopy equivalence. If $m=$ 2, as $Y=X_{1} \cap X_{2}$ is contractible, $X / Y=X_{1} / Y \cup X_{2} / Y$ and $X_{1} / Y \cap X_{2} / Y=Y / Y$ is a point, the lemma follows easily in this case.

Assume the lemma holds for $<m$. Then $X_{1} \cap\left(\bigcup_{i>1} X_{i}\right)=\bigcup_{i>1}\left(X_{1} \cap X_{i}\right)$ is homotopy equivalent to $\bigvee_{i>1}\left(X_{1} \cap X_{i}\right)$ by induction and hence contractible. Therefore $X$ is homotopy equivalent to $X_{1} \vee\left(\bigcup_{i>1} X_{i}\right)$, which is in turn homotopy equivalent to $X_{1} \vee X_{2} \vee \cdots \vee X_{m}$.

We refer readers who are unfamiliar with the geometry of groups of Lie type and the theory of Coxeter groups to [A] or [B]. From time to time by abuse of notation we identify an element of $N$ with its image in the Weyl group $W=N / H$. We may also use the same notation for a simplex of a complex and the subcomplex consisting of its faces.

Lemma 2. Let $W_{J}=G_{J} \cap N / H$ be a parabolic subgroup of $W$, where $G_{J}=$ $\bigcap_{j \in J \subseteq I} G_{i}$. Then each coset $W_{J} d$ has a unique element $d_{J}$ of minimal length such that $l\left(w d_{J}\right)=l(w)+l\left(d_{J}\right)$ for all $w \in W_{J}$. Here $l$ is the length function on $W$ with respect to $S$.

Proof. This is exercise 3 , page 37 in [B].

Define a partial ordering $\leqslant$ on $W$ by $v \leqslant w$ if and only if $w=u v$ and $l(w)=$ $l(u)+l(v)$ for some $u \in W$. This is a well defined ordering, and $w_{0}$, the longest element of $W$, is the unique maximal element with respect to this order. See for instance exercise 6 , page 155 in $[\mathrm{A}]$.

Lemma 3. Let $\Phi$ be the Coxeter complex (see page 210 in $[\mathrm{A}]$ for the definition) for $W, \mathbf{C} \in \Phi$ the fundamental chamber and $\sigma \in \Phi$ a simplex. Then there is a unique $w_{\sigma} \in W$ with $\sigma \subseteq \mathbf{C} w_{\sigma}$ such that for any chamber $\mathbf{C} w$ containing $\sigma, w_{\sigma} \leqslant w$. Moreover, there is a sequence of chambers $\mathbf{C} w_{\sigma}=\mathbf{C} w_{1}, \mathbf{C} w_{2}, \ldots, \mathbf{C} w_{n}=\mathbf{C} w$, each containing $\sigma$, such that $w_{i} \leqslant w_{i+1}$ with $l\left(w_{i+1}\right)=l\left(w_{i}\right)+1,1 \leqslant i<n$.

Proof. This is a direct consequence of Lemma 2.

\section{Proof of the Theorem}

Let $\mathbf{C}=\left\{G_{i} ; 1 \leqslant i \leqslant n\right\}$ be the fundamental chamber of $\mathcal{B}$ and $\Sigma=\bigcup_{w \in W} \mathbf{C} w$. Then $\Sigma$ is an apartment stabilized by $N$ isomorphic to the Coxeter complex $\Phi$. Hence, as $\Phi$ has the homotopy type of a $(n-1)$-sphere,

Proposition 1. $\Sigma$ has the homotopy type of a $(n-1)$-sphere.

As $G=B N B=B N U$, it is obvious that

Proposition 2. $\mathcal{B}=\bigcup_{u \in U} \Sigma^{u}$, where $\Sigma^{u}=\bigcup_{w \in W} \mathbf{C} w u$.

Notice that for $u_{i} \in U, 1 \leqslant i \leqslant k, \bigcap_{1 \leqslant i \leqslant k} \Sigma^{u_{i}}$ is isomorphic to

$$
\left(\bigcap_{1 \leqslant i \leqslant k} \Sigma^{u_{i}}\right)^{u_{1}-1}=\bigcap_{1 \leqslant i \leqslant k} \Sigma^{u_{i} u_{1}^{-1}} .
$$

So by the argument in the introduction, a proof of the following proposition will complete the proof of the Solomon-Tits Theorem.

Proposition 3. For distinct $u_{1}, u_{2}, \ldots, u_{k} \in U-\{1\}, k \geqslant 1, \Sigma \cap \cap \Sigma^{u_{k}}$ is contractible. 
The rest of paper is dedicated to the proof of Proposition 3. For simplicity, we deal only with the case $k=1$. The same proof works for $k>1$.

Lemma 4. (1) For $1 \neq u \in U, \mathcal{T}=\Sigma \cap \Sigma^{u}$ is the subcomplex of $\Sigma$ consisting of simplices fixed by $u$. Moreover, $\mathcal{T}$ is a union of chambers.

(2) If $\mathbf{C} w \in \mathcal{T}$ and $v \leqslant w$, then $\mathbf{C} v \in \mathcal{T}$.

Proof. Let $\mathbf{C}_{J}=\left\{G_{j} ; j \in J \subseteq I\right\} \subseteq \mathbf{C}$ and assume $\mathbf{C}_{J} v_{1} u=\mathbf{C}_{J} v_{2}$ for some $v_{1}, v_{2} \in W$. Then $G_{J} v_{1} u=G_{J} v_{2}$. As $N \cap G_{J}=N_{J}$ is the preimage of $W_{J}$ in $N$, we may choose $v_{1}$ and $v_{2}$ as in Lemma 2. Let $l\left(v_{k}\right)=r_{k}, k=1,2$. Without loss assume $r_{1} \leqslant r_{2}$. Otherwise we consider $u^{-1}$ instead. Set $v_{1}=x_{1} x_{2} \ldots x_{r_{1}}$ with $x_{k}$ 's from $S$. As $s B w \subseteq B w B \cup B s w B$ for $s \in S$ and $w \in W$, we have

$$
v_{1} u v_{2}^{-1} \in B v_{2}^{-1} B \cup \bigcup_{1 \leqslant k_{1}<k_{2}<\cdots<k_{t} \leqslant r_{1}} B x_{k_{1}} x_{k_{2}} \ldots x_{k_{t}} v_{2}^{-1} B .
$$

Then as $v_{1} u v_{2}^{-1} \in G_{J}=B N_{J} B$, either $v_{1} u v_{2}^{-1} \in B v_{2}^{-1} B \subseteq G_{J}$ or there are $1 \leqslant k_{1}<k_{2}<\cdots<k_{t} \leqslant r_{1}$ such that $v_{1} u v_{2}^{-1} \in B x_{k_{1}} \ldots x_{k_{s}} v_{2}^{-1} B \subseteq G_{J}$. In the first case, $v_{2} \in G_{J}$ and by Lemma $2 v_{2}=1$. Consequently $v_{1} \in G_{J} v_{2} u^{-1}=G_{J}$, and again by Lemma $2 v_{1}=1$. In the second case, set $v_{0}=x_{k_{1}} \ldots x_{k_{t}}$. Then $v_{0} \in G_{J} v_{2}$ with $l\left(v_{0}\right) \leqslant t \leqslant r_{1} \leqslant r_{2}=l\left(v_{2}\right)$; hence by Lemma 2 , all equalities must hold and $t=r_{1}, r_{1}=r_{2}, v_{0}=v_{1}=v_{2}$. Therefore $v_{1} u v_{2}^{-1} \in B v_{0} v_{2}^{-1} B=B$, i.e. $u \in B \cap B^{v_{1}}$. In either case, $v_{1}=v_{2}, u \in B \cap B^{v_{1}}$ and $\mathbf{C}_{J} v_{1} \subseteq \mathbf{C} v_{1} \subseteq \mathcal{T}$. Part (1) is proved.

For $v \leqslant w \in W$ and $\mathbf{C} w \in \mathcal{T}$, assume $w=x v$ with $l(w)=l(x)+l(v)$. Let $x=x_{1} x_{2} \ldots x_{r}$ with $r=l(x)$ and $x_{i} \in S$. For $t \leqslant r$, set $w_{t}=x_{t} \ldots x_{r} v$. Now for any $s \in S$ and any $w \in W$ with $s w \leqslant w$, we have $B \cap B^{w} \leqslant B^{s w}$. Applying the observation to $x_{1}$ and $w$, we have $u \in B \cap B^{w} \in B^{w_{2}}$, so $\mathbf{C} w_{2} \in \mathcal{T}$. Now part (2) follows inductively.

Lemma 5. $\mathcal{T}$ is contractible.

Proof. As $\mathbf{C} \in \mathcal{T}, \mathcal{T}$ is nonempty. $\mathcal{T}$ is connected by Lemma 3 and Lemma 4.2. Recall that $w_{0}$ is the longest element in $W$. Notice that $\mathbf{C} w_{0} \notin \mathcal{T}$, as $u \notin B \cap B^{w_{0}}=$ $H$.

Pick a $\bar{w} \in W$ with $\mathbf{C} \bar{w} \subseteq \mathcal{T}$ such that $l(\bar{w})$ is maximal. Set

$$
\mathcal{T}_{1}=\bigcup_{w \neq \bar{w}, u \in B \cap B^{w}} \mathbf{C} w \subseteq \mathcal{T} .
$$

For a simplex $\sigma \in \mathcal{T}_{1} \cap \mathbf{C} \bar{w}$, choose $v \in W$ as in Lemma 3 such that $\sigma \in \mathbf{C} v$. Then by Lemma $3, v \leqslant \bar{w}$. So $\mathbf{C} v \in \mathcal{T}$ by Lemma 4.2. But $\sigma \in \mathcal{T}_{1}$, and consequently there is a $w \in W$ with $\sigma \in \mathbf{C} w \in \mathcal{T}_{1}$. This forces $v \neq \bar{w}$ and $\mathbf{C} v \in \mathcal{T}_{1}$. Therefore by Lemma 3 there is an $s \in S$ with $v \leqslant s \bar{w} \leqslant \bar{w}$ and $\sigma \in \mathbf{C} s \bar{w} \subseteq \mathcal{T}_{1}$. So $\sigma \subseteq \mathbf{C} \bar{w} \cap \mathbf{C} s \bar{w}$, an $(n-2)$-simplex of $\mathbf{C} \bar{w}$.

On the other hand, for any $s \in S$ with $s \bar{w} \leqslant \bar{w}$, it is obvious that $\mathbf{C} s \bar{w} \in \mathcal{T}_{1}$ and $\mathbf{C} \bar{w} \cap \mathbf{C} s \bar{w} \subseteq \mathcal{T}_{1} \cap \mathbf{C} \bar{w}$. We have shown that $\mathcal{T}_{1} \cap \mathbf{C} \bar{w}$ is the union of $(n-2)$ simplices $\mathbf{C} \bar{w} \cap \mathbf{C} s \bar{w}$ of $\mathbf{C} \bar{w}$ with $s \bar{w} \leqslant \bar{w}$. As $\bar{w} \neq w_{0}$, there is an $s \in S$ with $s \bar{w}>\bar{w}$. Therefore there are at most $n-1(n-2)$-simplices of $\mathbf{C} \bar{w}$ in $\mathcal{T}_{1}$, and their intersection contains a vertex; hence $\mathcal{T}_{1} \cap \mathbf{C} \bar{w}$ is contractible. Applying Lemma 1 , we conclude that $\mathcal{T}$ is homotopy equivalent to $\mathcal{T}_{1}$.

Notice that $\mathcal{T}_{1}$ is also a union of chambers and satisfies Lemma 3, part (2). Therefore, repeating the above procedure gives that $\mathcal{T}$ is homotopy equivalent to $\mathbf{C}$, the fundamental chamber, hence contractible. 


\section{REFERENCES}

[A] M. Aschbacher, Finite Group Theory, Cambridge University Press, Cambridge,1986. MR 95b:20002

[B] Bourbaki, N., Groupes et algebras de Lie, 4, 5, 6, Masson, Paris, 1981. MR 83g:17001

[CL] C.W. Curtis and G. I. Lehrer, A new proof of a theorem of Solomon-Tits, Proc. of AMS v. 82, No. 2 (1982) 154-156. MR 83j:20048

[G] H. Garland, $p$-adic curvature and the cohomology of discrete subgroups of $p$-adic groups, Ann. Math.(2) 97 (1973) 375-393. MR 47:8719

[S] L. Solomon, The Steinberg character of a finite group with a $B N$-pair, Theory of Finite Groups, R. Brauer and C.H. Sah, Eds., Benjamin, New York, 1969, pp. 213-221. MR 40:220

Department of Mathematics, Caltech, Pasadena, California 91125

E-mail address: chaoku@cco.caltech.edu 